\title{
Quality of acute coronary care in emerging economies
}

\author{
Martin Bobak MD, Harry Hemingway MB BChir MSc
}

$\infty$ See related research paper by Tatu-Chitoiu and colleagues, page 1207

$\mathrm{R}$ easons for geographical and temporal variations in the death rates from coronary artery disease are not clear. To date, most explanations have focused on factors that affect the incidence of coronary artery disease, such as smoking, alcohol, diet and psychosocial factors. ${ }^{1}$ Because of a lack of high-quality registry data from many populations, much less attention has been paid to factors, such as the quality of medical care, that may influence survival.

In this issue, Tatu-Chitoiu and colleagues ${ }^{2}$ report the inhospital mortality among more than 9000 patients admitted to hospital in Romania with acute ST-elevation myocardial infarction. The authors report a low rate of use of types of care (e.g., thrombolysis) that have long been the standard in Western countries and very low rates of use of newer standards of care (e.g., primary percutaneous coronary intervention). ${ }^{2}$ In this study, women received such care less often than men and had longer times between the onset of symptoms and presentation to hospital. The in-hospital mortality rate for patients with acute ST-elevation myocardial infarction in this study was $12.7 \%$. Tatu-Chitoiu and colleagues compared this rate with that from the Euro Heart Survey $(<7 \%)$ and the US National Registry of Myocardial Infarction $(8 \%)$. The authors conclude that, in Romania, inadequate acute medical care contributed not only to the high inhospital mortality but also to the overall mortality from coronary artery disease.

However, important caveats need to be considered when comparing the in-hospital mortality between Romania and Western countries, because the patient populations may not be comparable. First, in-hospital mortality depends on length of hospital stay, which might be expected to be longer in Romania than in the West. Second, data from the Western studies represent a selected group of hospitals (and for the European data, selected patients), which makes it difficult to generalize the results to whole countries. Finally, the phenotypes and case mixes may not be comparable between populations, because of the presence of other comorbidities or differences in diagnostic practice (e.g., the use of troponins).

However, these registry data are important both nationally and internationally. For Romania, the comparison to international data provides a strong stimulus to improve the provision of care. Other countries can benefit from these data because most lack national registries. Only the United Kingdom $^{3}$ and Sweden ${ }^{4}$ are currently developing national registries with consecutive patients from more than $95 \%$ of hospitals. As more countries develop registries, international scrutiny of the many factors, not just quality of care, that affect disease outcomes will provide insight into the improvement of these outcomes. Currently, programs that study the

\section{Key points}

- Data from the Romanian registry of myocardial infarction show that the quality of care is low and that the in-hospital case-fatality rates are high.

- Comparisons between Romania and Western countries are hampered by different registry designs and different lengths of hospital stay.

- The effects of quality of care on case-fatality rates of coronary artery disease in the general population remain inconclusive.

- Registries for coronary artery syndromes with standardized protocols in both developed and developing countries would foster international comparisons.

prognosis of diseases are much less well developed than those that study onset. ${ }^{5}$

The data about the effects of quality of care on casefatality rates of coronary artery disease in the general population are inconclusive. The World Health Organization's MONICA Project ${ }^{6}$ provides one of the few robust international comparisons of outcomes in acute myocardial infarction. This project included an assessment of 30-day casefatality rates (thus avoiding the biases of in-hospital mortality) in 31 countries, including 8 in Central and Eastern Europe, between the mid-1980s and mid-1990s. Although medical care has changed markedly since this project was carried out, it highlights challenges for future research. Ecological cross-sectional analyses of the MONICA data suggest that the quality of care contributed to the high mortality from coronary artery disease in Eastern Europe. ${ }^{1}$ However, in these analyses, it was difficult to separate the effects of care from those of risk factors for coronary artery disease and socio-economic characteristics.

Detailed analyses of the 10-year changes in mortality and case-fatality rates from coronary disease in relation to the quality of care in the 31 populations with good quality data in the MONICA study were also ambiguous. ${ }^{7}$ Improvements in the quality of coronary care correlated well with declines in 28-day case-fatality rates. However, improvements in coronary care were associated more strongly with pre-hospital mortality than with in-hospital mortality.

This counterintuitive finding suggests unmeasured confounding. Improvements in coronary care and the rates of coronary artery disease, both over time and across populations, may be associated with unmeasured factors, possibly related to social and economic factors that may influence the severity of disease. For example, gross national income was inversely associated with 30-day mortality in 53 countries. $^{8}$

Martin Bobak and Harry Hemingway are with the Department of Epidemiology and Public Health, University College London, London, UK. 
The pathways leading to improved coronary disease outcomes may include, for example, dietary habits and and health behaviours, as well as primary and secondary prevention. In the case of Romania, it would be interesting to examine the levels and trends in pre-hospital mortality and how they compare with the levels and trends in other populations.

A related important question, although beyond the scope of the study by Tatu-Chitoiu and colleagues, is whether changes in the quality of acute coronary care are associated with declining rates of death from coronary artery disease. Modelling based on coefficients derived from clinical trials suggests that much of the temporal decline in population rates of mortality from coronary artery disease can be attributed to improvements in acute coronary care and secondary prevention. ${ }^{9}$ However, direct evidence is sparse. Data from the MONICA study show robust correlations between changes in coronary care, case-fatality rates and mortality rates from coronary artery disease, ${ }^{7}$ but the data support the conclusion that incidence is the major determinant of the overall mortality rates from coronary artery disease. ${ }^{10}$

Worldwide, the care of most patients with myocardial infarction currently occurs behind closed doors, and data are not available for national or international comparisons. The paper by Tatu-Chitoiu and colleagues is an uncommon and important example of how high-quality registry data can identify areas for change. Although more registries are being established, perhaps some of the MONICA registries in Eastern Europe and elsewhere could be revitalized. If this occurs, elements of data collection, including followup protocols and diagnostic criteria (e.g., genetic and biomarker data), need to be standardized to challenge existing practices and guide new ones, as well as to link etiologic and prognostic research.

\section{Competing interests: None declared.}

Contributors: Both of the authors designed the commentary. Martin Bobak drafted the first version, and Harry Hemingway expanded it and revised it critically. Both authors approved the final version submitted for publication.

\section{REFERENCES}

1. Bobak M, Marmot M. Coronary heart disease in Central and Eastern Europe and the former Soviet Union. In: Marmot M, Elliott P, editors. Coronary heart disease epidemiology. From aetiology to public health. 2nd ed. Oxford (UK): Oxford University Press; 2005. p. 83-101.

2. Tatu-Chitoiu G, Cinteza M, Dorobantu M, et al. In-hospital case fatality rates of acute myocardial infarction in Romania. CMAJ 2009;180:1207-12.

3. Birkhead JS, Weston C, Lowe D. Impact of specialty of admitting physician and type of hospital on care and outcome for myocardial infarction in England and Wales during 2004-5: observational study. BMJ 2006;332:1306-11.

4. Stenestrand U, Lindbäck J, Wallentin L, et al. Long-term outcome of primary percutaneous coronary intervention vs prehospital and in-hospital thrombolysis for patients with ST-elevation myocardial infarction. JAMA 2006;296:1749-56.
5. Hemingway H. Prognosis research: why is Dr. Lydgate still waiting? J Clin Epidemiol 2006;59:1229-38.

6. World Health Organization. The WHO MONICA project. The Organization; 2008. Available: www.ktl.fi/monica (accessed 2009 Apr. 23).

7. Tunstall-Pedoe H, Vanuzzo D, Hobbs M, et al. Estimation of contribution of changes in coronary care to improving survival, event rates, and coronary heart disease mortality across the WHO MONICA Project populations. Lancet 2000;355:688-700.

8. Orlandini A, Díaz R, Wojdyla D, et al. Outcomes of patients in clinical trials with ST-segment elevation myocardial infarction among countries with different gross national incomes. Eur Heart J 2006;27:527-33.

9. Unal B, Critchley JA, Capewell S. Explaining the decline in coronary heart disease mortality in England and Wales between 1981 and 2000. Circulation 2004;109:1101-7.

10. Tunstall-Pedoe H, Kuulasmaa K, Mähönen M, et al. Contribution of trends in survival and coronary-event rates to changes in coronary heart disease mortality: 10year results from 37 WHO MONICA project populations. Monitoring trends and determinants in cardiovascular disease. Lancet 1999;353:1547-57.

Correspondence to: Prof. Martin Bobak, Department of Epidemiology and Public Health, University College London, 1-19 Torrington Pl., London, WC1E 6BT, United Kingdom; fax 44207813 0242; m.bobak@ucl.ac.uk
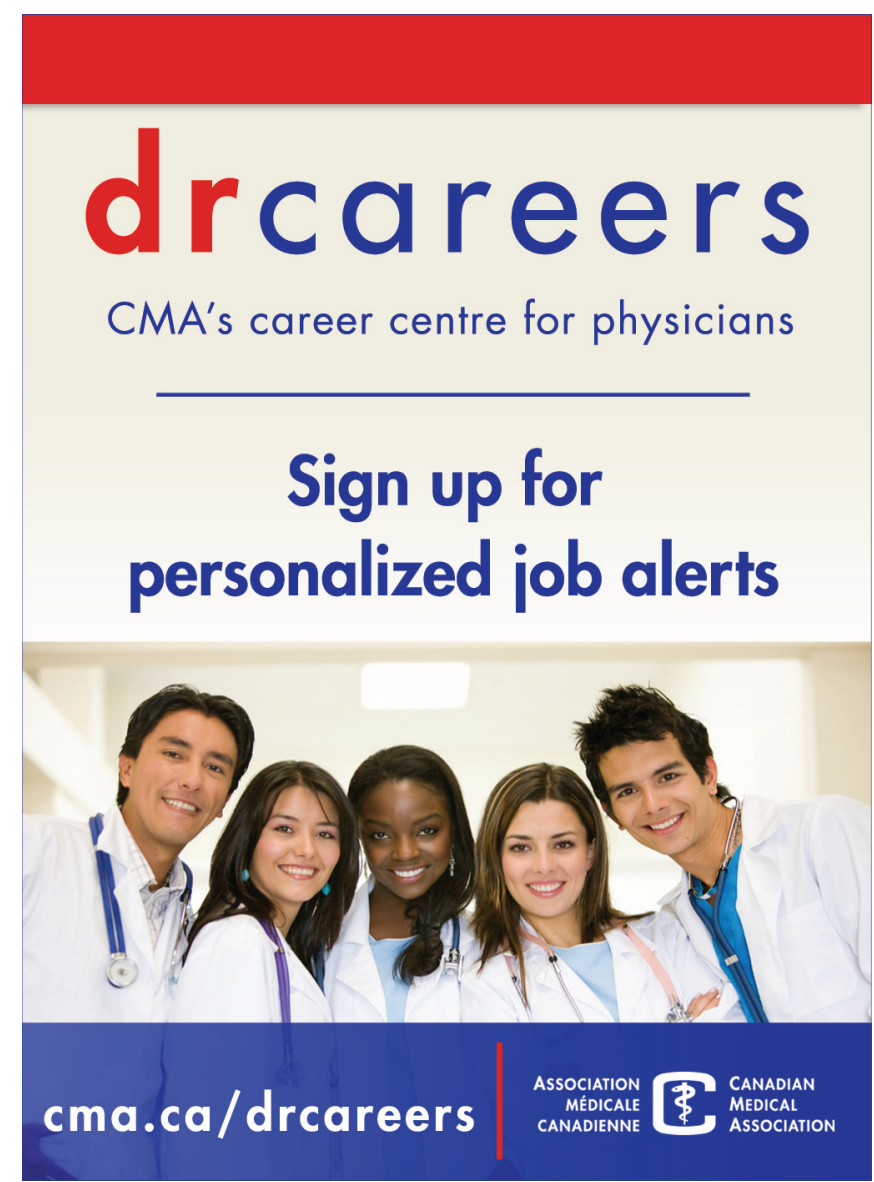